\title{
Data-driven Nonlinear Prediction Model for Price Signals in Demand Response Programs
}

De Zotti, Giulia; Binder, Hanne ; Hansen, Anders Bavnhøj; Madsen, Henrik; Relan, Rishi

Published in:

Proceedings of Probabilistic Methods Applied to Power Systems 2020

Publication date:

2020

Document Version

Peer reviewed version

Link back to DTU Orbit

Citation (APA):

De Zotti, G., Binder, H., Hansen, A. B., Madsen, H., \& Relan, R. (2020). Data-driven Nonlinear Prediction Model for Price Signals in Demand Response Programs. In Proceedings of Probabilistic Methods Applied to Power Systems 2020 IEEE.

\section{General rights}

Copyright and moral rights for the publications made accessible in the public portal are retained by the authors and/or other copyright owners and it is a condition of accessing publications that users recognise and abide by the legal requirements associated with these rights.

- Users may download and print one copy of any publication from the public portal for the purpose of private study or research.

- You may not further distribute the material or use it for any profit-making activity or commercial gain

- You may freely distribute the URL identifying the publication in the public portal 


\section{Data-driven Nonlinear Prediction Model for Price Signals in Demand Response Programs}

\author{
Giulia De Zotti \\ Applied Mathematics and Computer Science \\ Technical University of Denmark \\ Lyngby, Denmark \\ gizo@dtu.dk
}

\author{
Henrik Madsen \\ Applied Mathematics and Computer Science \\ Technical University of Denmark \\ Lyngby, Denmark \\ hmad@dtu.dk
}

\author{
Hanne Binder \\ Business Development and Support \\ Energinet \\ Fredericia, Denmark \\ hbr@energinet.dk
}

\author{
Anders Bavnhøj Hansen \\ Business Development and Support \\ Energinet \\ Fredericia, Denmark \\ abh@energinet.dk
}

\begin{abstract}
In power systems, electrical consumers can become a significant source of flexibility, by adjusting their consumption according to grid's needs while respecting their operational constraints. Consumers' flexibility potential can be exploited through the submission of dynamic electricity prices. Such prices are able to describe the variable condition of the power system and are broadcast to the consumers in order to obtain a certain change in consumption. The formulation of effective dynamic prices requires the development of proper models that describe the price responsiveness of electrical consumers. In this paper, we propose a nonlinear prediction model for the dynamic electricity prices in demand response (DR) programs. Specifically, the nonlinear autoregressive with exogenous input (NARX) model structure is used to learn from available data to predict appropriate electricity price signals. For the validation of the model (in an aggregate manner) in predicting consumers' price-response, the data from 10 Danish households is utilised, which has provided by the Danish Transmission Service Operator (TSO) Energinet.
\end{abstract}

Index Terms-Demand response, Smart grid, Neural network, Electricity prices

\section{INTRODUCTION}

Demand response (DR) consists of changes in electricity usage by consumers from their normal consumption patterns [1]. DR programs are becoming a popular solution to address the balancing issues of stochastic generation from renewable energy sources (RES) in power systems [2]. In fact, they allow electricity generation and consumption to be balanced dynamically in real-time thereby improving the overall power system operation. The functioning of DR programs varies widely in their applications depending on incentives, e.g., contracts or dynamic prices.

In incentive-based DR programs, consumers delegate their consumption scheduling to an external agent that is entitled

The work has been partly funded by the Innovation Fund Denmark project CITIES (1305-0027B) and the European Union's Horizon 2020 Smart-Net project (grant agreement No 691405). The work by Henrik Madsen was partly supported by the Flexible Energy Denmark and the Center Denmark projects.

978-1-7281-2822-1/20/\$31.00 @2020 IEEE

\author{
Rishi Relan \\ Applied Mathematics and Computer Science \\ Technical University of Denmark \\ Lyngby, Denmark \\ risre@dtu.dk
}

to directly control the consumers' loads. Such programs rely on a two-way communication scheme, where consumers' appliances receive a signal from an aggregator to change their consumption and they inform the aggregator with the new amount of consumption. Incentive-based DR programs require consumers to lose part of their autonomy, leading to privacy issues. Therefore, these programs find only limited participation from consumers. To overcome such an issue, in incentive-based DR programs, dedicated markets can be designed (i.e., the ancillary services market). The proposed market structures mainly use linear and deterministic bidding and clearing process [3]. Nevertheless, the increasing level of uncertainty in consumption and generation necessitates the adoption of nonlinear and stochastic operation methods [4].

In dynamic prices-based schemes, each consumer receives a time-varying price signal by the home energy management system (HEMS) (i.e., local controller) to schedule internal generation and consumption aiming to minimise the overall cost. In this setting, individual feedback from consumers is not required and operators can only see the aggregate consumers' reaction to prices at power systems level [5]. Moreover, by approaching consumers as an aggregate, there is no operational nor computational limit to the number of households involved in such dynamic prices-based schemes [6]. Although dynamic price-based DR programs neither affect consumers' autonomy nor privacy, they expect DR operators to formulate electricity price signals. By influencing consumers' aggregate priceresponse, dynamic prices might also affect power system' security [6]. Therefore, a robust price generating model considering heterogeneous consumers' dynamics and different DR capabilities needs to be developed [7].

The technical literature proposes different linear modelling approaches to predict price signals, from linear regression [8] to time-series models [9], such as the auto regressive (AR) and the auto-regressive moving average (ARMA) models [10], which cannot always describe the often non-linear behaviour 
of price-response.

However, in recent years, various artificial neural network (ANN) configurations have been evaluated to forecast the short-term electricity prices due to their capability of inferring hidden nonlinear relationship in the data. In [11], the authors discussed the effectiveness of an ANN when forecasting nonspiky prices. In [12], the day-ahead electricity prices were forecasted by knowing the day of the week, the estimated electricity demand, historical electricity prices and time slot of the day. However, such studies mainly applied ANN as a predictor to improve the profitability of the utility investments [13], omitting any analysis of the factors affecting consumers' flexibility.

In this paper, we propose a nonlinear auto-regressive with exogenous inputs (NARX) model to predict dynamic electricity prices. The main objective of this study is to learn consumers' flexibility behaviour in relation to different factors. Such an understanding can be exploited by a DR operator to formulate adequate electricity prices that can achieve a certain change in load under dynamic prices-based schemes.

An NARX recurrent ANN model structure is trained based on the attributes/features extracted from the data provided by the Danish TSO Energinet [14]. The main contribution of this work is the adoption of an NARX model for the generation of dynamic electricity prices in DR programs. To the best of authors' knowledge, this is the first instant where a nonlinear model is used to formulate the dynamic electricity prices that induce a certain change in consumption from flexible consumers. The rest of the paper is structured as follows: in Section II the problem is formulated; in Section III, the ANN modelling is described; in Section IV, the numerical analysis is provided; finally, Section $\mathrm{V}$ summarises the main findings and includes the conclusions.

\section{Case Study Model Definition}

In this section, we explain the problem considered in this study. Specifically, we are interested in generating proper dynamic electricity prices for price-based DR programs.

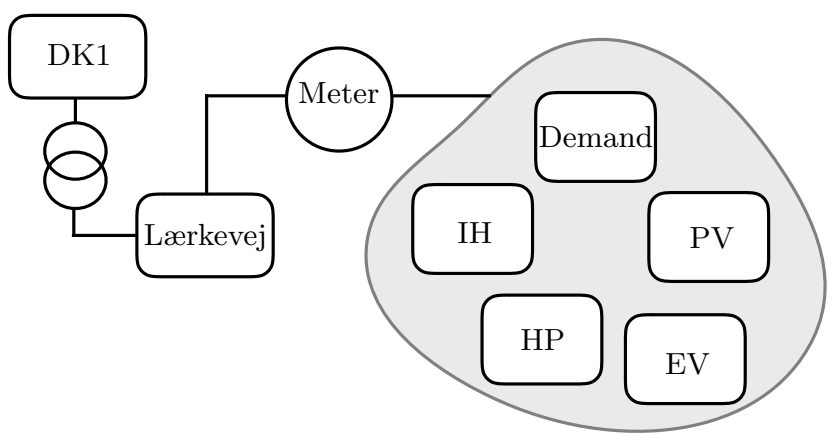

Fig. 1. Simulation setting for the case of Lærkevej [15]. DK1 consists of the Danish transmission area; the meter is placed at the level of the feeder. The electricity demand of each household includes: immersion heater (IH), photovoltaic (PV) panel, electric vehicle (EV), heat pump (HP) and a certain inflexible household electricity demand.

To generate suitable price signals, we need to have information about consumers' aggregate price-response. As we deal with a one-way communication scheme where no individual feedback is provided by individual consumers, operators need to deal with aggregate measurements of consumers' priceresponse. However, a major challenge is the heterogeneity of consumers' loads, which might follow different dynamics and can have different constraints. For this reason, we study the price-response of a pool of consumers characterised by various types of loads.

Fig. 1 presents the case study considered in this paper: Lærkevej (i.e., "Lark street"), consisting of a typical street in Denmark with 10 different houses. In Lærkevej, each house is equipped with a heat-pump (HP), a photovoltaic panel (PV), an immersion heater (IH) and an electric vehicle (EV). Furthermore, each house is characterised by a certain inflexible household electricity demand. Lærkevej is connected to DK1 transmission area of the Danish power system through a feeder. To have an aggregate information about consumers' price-response in Lærkevej, we deal with electricity demand measurements at the feeder level.

In order to generate the DR data necessary for the estimation of a nonlinear electricity price model, the configuration of Lærkevej was simulated through the long-term energy planning tool SIFRE (SImulation of Flexible and Renewable Energy sources) at Energinet [16]. Specifically, the SIFRE tool can be used to schedule consumers' flexible consumption aiming to achieve cost minimisation. For this case study, the SIFRE tool was used to optimise the electricity consumption of 10 houses over seven days, assuming perfect knowledge for every useful parameter (i.e., outdoor temperature, dynamic electricity price, solar irradiation and so on). The electricity consumption data generated using the SIFRE tool represents the aggregate consumers' price-response.

\section{NONLINEAR PRICE MODELling}

In this section, first we describe briefly the NARX model structure and its application for modelling the dynamic electricity price signals for DR programs. The dynamic electricity prices are expected to address a certain change in the aggregate electricity consumption. Therefore, it is important to investigate the effect of various factors such as the outdoor temperature, electricity price, outdoor temperature, type of the day, physical capability of the grid to satisfy a certain demand (i.e., events of bottleneck and congestion status), time and distributed energy generation (e.g., PV installations) on the price-response.

\section{A. NARX Model Structure}

It is common to observe the long-term dependence in the time-series data (e.g., electricity price, electricity load timeseries) observed in the real-world power systems. To model such real-world time series, the recurrent ANN are commonly used. The NARX model structure is a specific kind of recurrent ANN which can be used to efficiently model a time-series with 
long-term dependencies. In discrete-time the NARX model structure can be described as [17]:

$$
\begin{aligned}
y(n+1) & =\mathcal{F}\left[y(n), \ldots, y\left(n-d_{y}+1\right) ;\right. \\
& \left.u(n), u(n-1), \ldots, u\left(n-d_{u}+1\right)\right], \\
& =\mathcal{F}[y(n) ; u(n)],
\end{aligned}
$$

where $u(n)$ and $y(n)$ denote, respectively, the input and the output of the model at discrete time $n$. The parameters $d_{u} \geq 1$ and $d_{y} \geq 1, d_{u} \leq d_{y}$, are memory delays. The unknown nonlinear function $\mathcal{F}(\bullet)$ can be approximated by a MultiLayer Perceptron (MLP) [17], [18]. There are mainly two modes to train a NARX network:

- Series-Parallel (SP) Mode: In this case, the output regressor of the NARX-network is formed by the measured output values and can be written as:

$$
\begin{aligned}
\hat{y}(n+1) & =\hat{\mathcal{F}}\left[y_{S P}(n), u(n)\right] \\
& =\left[y(n), \cdots, y\left(n-d_{y}+1\right)\right. \\
& \left.u(n), u(n-1), \cdots, u\left(n-d_{u}+1\right)\right]
\end{aligned}
$$

- Parallel $(P)$ Mode: In this case, the estimated outputs are fed-back and the output regressor can be defined as [19]:

$$
\begin{aligned}
\hat{y}(n+1) & =\hat{\mathcal{F}}\left[y_{P}(n), u(n)\right] \\
& =\left[\hat{y}(n), \cdots, \hat{y}\left(n-d_{y}+1\right)\right. \\
& \left.u(n), u(n-1), \cdots, u\left(n-d_{u}+1\right)\right],
\end{aligned}
$$

It is worth noting that the feedback mechanism is present only in the P-Mode. The basic fundamental building block of an NARX neural network is an artificial neuron, that receives inputs, modifies its internal state and produces outputs. The output $y_{n n}$ of a neuron in a network can be formulated as:

$$
y_{n n}=f\left(\sum_{i=1}^{d}\left(w_{i}^{T} u_{i}\right)+\beta_{\mathbb{N}}\right), \quad i=1, \ldots, d
$$

where $f(\bullet)$ is the nonlinear activation function, $u_{i} \in U \in \mathbb{R}^{d}$ is the training input to the neuron, $w_{i} \in W \in \mathbb{R}^{d}$ is the weight of each input and $\beta_{\mathbb{N}}$ is the bias term [20]. The discriminant power of a single neuron can further be increased by applying a set of fixed nonlinear transforms $\phi(u)_{i}$ to the input vector $U$.

$$
y_{n n}=f\left(\sum_{i=1}^{d}\left(w_{i}^{T} \phi_{i}(u)\right)+\beta_{\mathbb{N}}\right), \quad i=1, \ldots, d
$$

- Similarly, for a feed-forward network containing ddimensional inputs, $\mathrm{M}$ hidden layer neurons and $\mathrm{K}$ outputs, the output vector of the network can be written as below:

$y_{n n_{K}}=g\left(\sum_{j=1}^{M}\left(w_{k j}^{(2)}\right)^{T} f\left(\sum_{i=1}^{d}\left(\left(w_{j i}^{(1)}\right)^{T} \phi_{i}(u)\right)+\beta_{1 \mathbb{N}}\right)+\beta_{2 \mathbb{N}}\right)$,

where $i=1, \ldots, d, j=1, \ldots, M, k=1, \ldots, k$ and the superscript $w^{(\bullet)}$ indicates the number of the network layer to which the corresponding weights matrix is associated. Moreover, $f$ and $g$ are the nonlinear activation functions related to the input and the hidden layer of the network respectively. Finally, $\beta_{1 \mathbb{N}}$ and $\beta_{2 \mathbb{N}}$ represent the bias terms added to the hidden layer and the output layers, respectively.

Depending on the complexity of the problem at hand, the most suitable number of neurons and hidden layers can widely vary. For a regression problem, in a multilayered neural network, the neurons learn the relationships between inputs and outputs in a training process by tuning biases and weights through a chosen algorithm which minimises the mean squared error (MSE) between the measured and predicted outputs which can be written as [21], [22]:

$$
J(\Theta)=\frac{1}{N} \sum_{i=1}^{N}\left\|y_{\text {Meas }}-\hat{y}_{N e t}\right\|^{2}
$$

where $N$ is the number of training samples, $\Theta$ is the set of parameters of the network (e.g. weights and biases) and $\hat{y}_{N e t}$ is the predicted output of the network [20].

Several optimisation algorithms such as Bayesian regularisation (BR) and Levenberg-Marquardt (LM) have been developed to minimise such a loss function. Depending upon the modelling task at hand, training data quality and the complexity of the problem, these optimisation algorithms vary in characteristics and performance. Although BR can identify very complex relationships among variables, it might not be able to reach convergence for big data-sets [23]. The LM algorithm gained popularity for its capability to achieve fast convergence in presence of big datasets [23], [24]. Therefore, we consider the LM training method and random sampling approach for training, validation and testing purposes.

The NARX neural network model structure formulated in this case study is a two-layer feed-forward network, where the hidden layer has a Sigmoid activation function and the output layer has a linear function. In this case study, various combination of the delay value and the number of neurons in the hidden layers have been investigated and the results are discussed in the following section.

\section{NUMERICAL ANALYSIS}

In this section, we investigate different NARX neural network models to describe the aggregate price-response of Lærkevej and design effective dynamic electricity prices considering the outdoor temperature, time, PV generation, electricity demand and congestion status as the input-data. For this study, the simulations in SIFRE are carried out considering a period of 3 years and hourly resolution. In Table I, the known parameters about Lærkevej are presented.

The outdoor temperature data is obtained from the historical values of Fredericia in 2017. When approaching the 3-year simulations in SIFRE, it is assumed that the outdoor temperature is constant over the years. Information about electricity demand is considered as aggregated for the 10 houses. The electricity price consists of the nodal price at Lærkevej, which therefore may depend on local electricity generation and congestion events. The nodal prices are calculated by the 
TABLE I

AVAILABLE INFORMATION ABOUT LÆRKEVEJ.

\begin{tabular}{|c|c|}
\hline Parameter & Unit \\
\hline Time & {$[-]$} \\
PV Generation & {$[\mathrm{MW}]$} \\
Electricity demand & {$[\mathrm{MW}]$} \\
Electricity price & {$[€ / \mathrm{MWh}]$} \\
Outdoor temperature & {$\left[{ }^{\circ} \mathrm{C}\right]$} \\
Congestion status & {$[-]$} \\
\hline
\end{tabular}

SIFRE tool, using the historical balancing prices in Denmark from year 2013 to 2015. The nodal prices at Lærkevej are shown in Fig. 2. Finally, from SIFRE, it is possible to know the congestion status at the feeder level. Specifically, it consists of a binary value that is 1 when an event of congestion occurs at the feeder of Lærkevej.

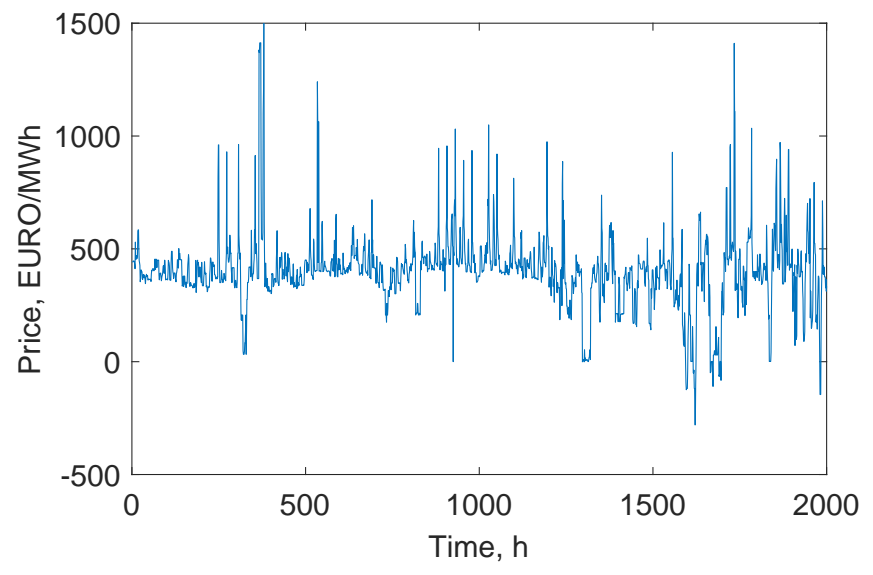

Fig. 2. Nodal electricity prices at Lærkevej.

\section{A. Data Pre-processing}

Before creating the ANN models, the heterogeneous information must be handled in a way that the data becomes comparable. In particular, the different data-sets must be normalised (i.e., outdoor temperature, electricity demand, time, PV generation). This way, we avoid excessively different magnitudes of different variables given to the ANN model. The normalisation is carried out by dividing each data-set by its highest value.

Electricity prices require particular attention. In fact, SIFRE has been developed in a way that it optimises the energy management of consumers over 7 days assuming perfect knowledge of the major factors for the duration of the simulation, and then running the simulation for several years by sliding the window forward. Also, it considers DR in a way that consumers are sensitive to the price variations to achieve absolute cost minimisation. Nevertheless, in SIFRE, consumers are not directly influenced by the absolute price magnitude but by the relative price difference. This condition embedded in SIFRE makes the overall reaction to prices highly dependant on the previous and future prices submitted to consumers. Therefore, the same price magnitude, in different optimisation time-frames, might lead to very different priceresponse reactions from the consumers. For this reason, the electricity price must be normalised differently than the other variables, normalising the values for each optimisation timeframe carried out in SIFRE (i.e., every 7 days). In Fig. 2 we show part of the electricity price-set (i.e., 2000 samples), as a compromise between graphical clarity and data completeness. The normalised electricity price-set is shown in Fig. 3.

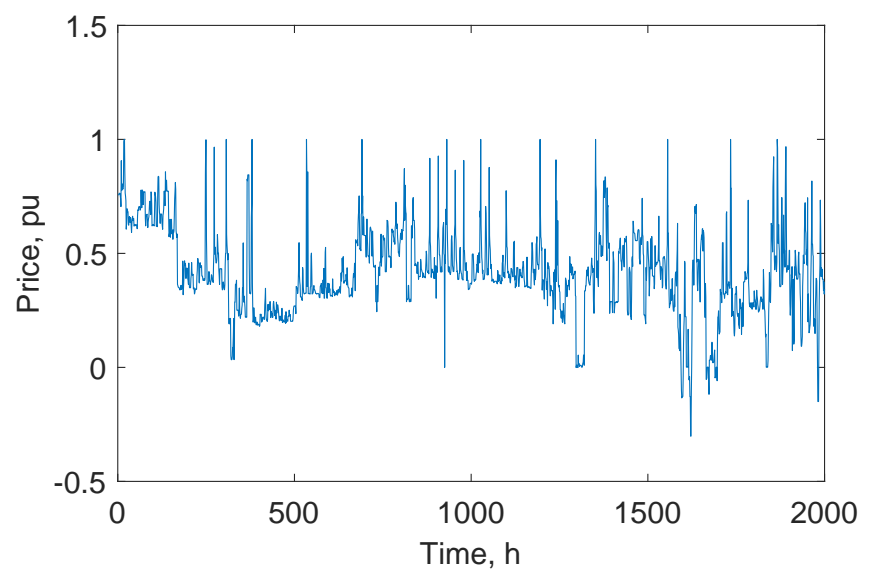

Fig. 3. Normalised nodal electricity prices at Lærkevej.

In this manner, excessive electricity peaks of Fig. 2 are avoided. In the following section, the ANNs are formulated and their performances are validated.

\section{B. Case Study}

In this subsection, we formulate ANN models using different assumptions regarding the input data. In particular, we formulate ANNs considering different levels of knowledge of the above discussed information about Lærkevej (i.e., time, PV generation, electricity demand, outdoor temperature and congestion status). Although most of this information is easily achievable at the system operator level (i.e., time, distributed generation, outdoor temperature, electricity demand and price), predicting the congestion of the system is not trivial, especially in hourly resolution at the feeder level. Moreover, it might become even more challenging when considering multiple feeders in the system. Therefore, we first model ANNs including information about congestion status. Afterwards, we repeat the study omitting this information.

Assumption 1: We assume to have perfect knowledge about the significant features of the system, including information about congestion status at the feeder level.

Remark 1: The reason is that we want to understand the significance of having full knowledge about the surrounding system in order to formulate accurate ANN models.

In the following, we formulate 5 different ANN models, whose performances are shown in Table II. In the simulations, each ANN is trained by dividing the data-set into three subsets (i.e., training, validation and testing set). Therefore, the model is tested to fit a certain portion of data. 
TABLE II

IN THE INPUT DATA-SET, IT IS INCLUDED: ELECTRICITY DEMAND, OUTDOOR TEMPERATURE, HOUR, PV GENERATION AND CONGESTION STATUS.

\begin{tabular}{|c|cc|ccc|c|}
\hline Model & $\begin{array}{c}\text { Hidden } \\
\text { neurons }\end{array}$ & $\begin{array}{c}\text { Delay } \\
\text { value }\end{array}$ & Train & $\begin{array}{c}\text { R } \\
\text { Valid }\end{array}$ & Test & $\begin{array}{c}\text { MSE } \\
\text { Test }\end{array}$ \\
\hline 1a & 2 & 5 & 0.872 & 0.875 & 0.872 & 0.0119 \\
2a & 5 & 10 & 0.892 & 0.893 & 0.891 & 0.0102 \\
3a & 15 & 10 & 0.898 & 0.887 & 0.896 & 0.0096 \\
$4 \mathrm{a}$ & 18 & 18 & 0.923 & 0.896 & 0.901 & 0.0093 \\
$5 \mathrm{a}$ & 30 & 30 & 0.933 & 0.888 & 0.883 & 0.0106 \\
\hline
\end{tabular}

In the simulations, we first approach a very simple case, considering 2 hidden neurons and a delay value of 5. Although this simple model has already quite good performance (as the multiple correlation coefficient, $\mathrm{R}$, of the testing data-set is $87.2 \%$ ), the results reported in the table show that the overall ANN performance improves when the complexity of the model increases. In fact, model $4 \mathrm{a}$ has the best performance, reaching a $\mathrm{R}$ value of $90.1 \%$. Moreover, this model shows the smallest MSE value. An example of the performance of model $4 \mathrm{a}$ is shown in Fig. 4. Nevertheless, when the model becomes excessively complex, no additional improvement is reached, as shown for the test period for model $5 \mathrm{a}$.

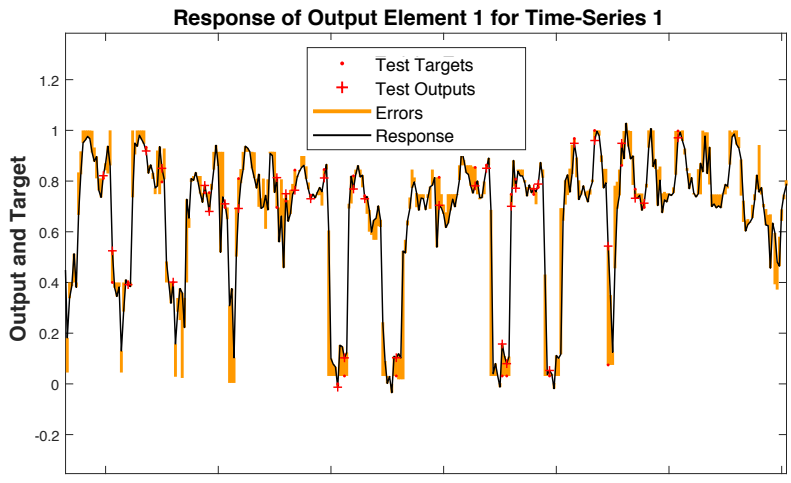

Fig. 4. Performance of the ANN model 4a.

In Fig. 4, the performance of an ANN model is shown graphically, plotting the targets (i.e., real data), the outputs (i.e., forecasted values from the ANN) and the prediction error.

The following part of this section discusses the design of the ANNs without the information about congestion status at the feeder level.

Assumption 2: We assume to have only limited knowledge about the significant features of the system. In particular, we omit the information about congestion status at feeder level as input data.

Remark 2: The reason is that such information can be difficult to predict in hourly resolution. Therefore, we want to approach the ANN formulation only considering information that can be easily obtained by the operator.

We carry out the simulations for the input data-set omitting the congestion status, whose results are reported in Table III.
TABLE III

IN THE INPUT DATA-SET, IT IS INCLUDED: TIME, ELECTRICITY DEMAND, OUTDOOR TEMPERATURE AND PV GENERATION.

\begin{tabular}{|c|cc|ccc|c|}
\hline Model & $\begin{array}{c}\text { Hidden } \\
\text { neurons }\end{array}$ & $\begin{array}{c}\text { Delay } \\
\text { value }\end{array}$ & Train & $\begin{array}{c}\text { R } \\
\text { Valid }\end{array}$ & Test & $\begin{array}{c}\text { MSE } \\
\text { Test }\end{array}$ \\
\hline 1b & 2 & 5 & 0.873 & 0.874 & 0.872 & 0.0115 \\
2b & 5 & 10 & 0.897 & 0.886 & 0.878 & 0.0113 \\
3b & 15 & 10 & 0.904 & 0.889 & 0.882 & 0.0112 \\
4b & 18 & 18 & 0.917 & 0.896 & 0.903 & 0.0090 \\
5b & 30 & 30 & 0.931 & 0.899 & 0.893 & 0.0102 \\
\hline
\end{tabular}

From the table, it can be seen that the performance of the ANN models are not compromised. On the contrary, dealing with a reduced input data-set leads to slightly better results. It is due to the fact that the congestion status is not an independent variable, as it depends on the overall PV generation and electricity demand (i.e., the overall power flow at the feeder). Fig. 5 shows the performance of the model $4 \mathrm{~b}$ for the modified data-set. It can be seen that model $4 \mathrm{~b}$ error has sharp peaks; this may be due to the absence of congestion signal whose effect is modelled as a switching (binary) signal in model $4 \mathrm{a}$ and needs to be further investigated.

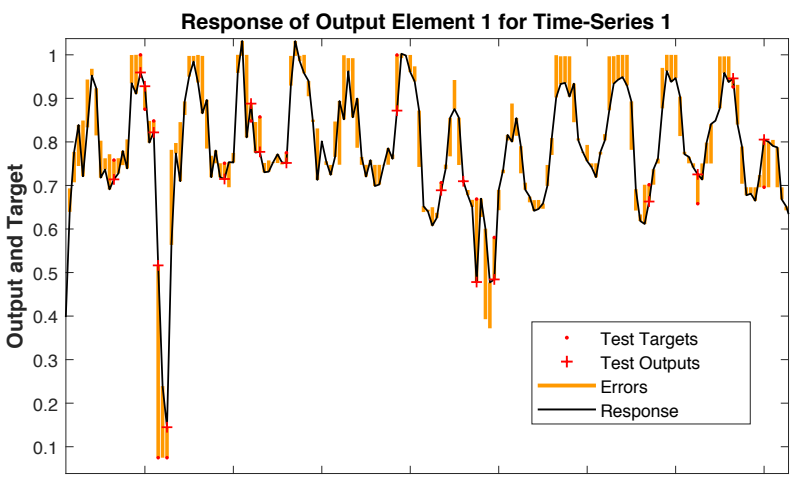

Fig. 5. Performance of the ANN model 4b.

\section{CONCLUSIONS}

This paper develops a nonlinear model to predict appropriate dynamic electricity prices with the application in DR programs. Several cases are discussed by considering different levels of knowledge about the significant features of the system. The simulations are based on data about the aggregated hourly consumption of 10 houses in Denmark, which have been provided by the Danish TSO Energinet.

This paper is conceptually divided into two main parts. In the first part, we presented and utilised the significant features of the system to design ANN models. Although having perfect knowledge about those features can facilitate the modelling of ANNs, some information might be difficult to collect in practice by the operator. In particular, knowledge about the congestion status is difficult to achieve in hourly resolution at the feeder level. Therefore, in the second part of this paper, we did not consider congestion' status and investigate whether the ANN performance is affected by that. From the numerical analysis, we realised that the performance is improved when 
disregarding congestion. Specifically, we showed that a relative simple ANN is able to achieve high predictability (i.e., a R value of $90.3 \%$ ) for the testing data-set. We can conclude that a single ANN is a promising tool to estimate the appropriate dynamic electricity price to alter consumers' behaviour, using estimated values of outdoor temperature, PV generation, time and electricity demand.

Although this study investigated consumers' price-response mainly in relation to prices variation (i.e., as considered in SIFRE), future studies are needed to consider price-response as sensitive to absolute price magnitude. Furthermore, a higher availability of real data about price-response of consumers will facilitate the formulation of more reliable models. Finally, future studies could be dedicated to investigate the price response of bigger pools of consumers.

\section{ACKNOWLEDGMENTS}

The authors would like to thank Hanne Storm Edlefsen, Thomas Sejr Jensen and Energinet for their availability. We are thankful to Prof. Juan Miguel Morales González and Dr. Ali Pourmousavi Kani for their constructive comments. Finally, we would like to thank the Danish Energy Agency, the Innovation Fund Denmark and the EU for the support.

\section{REFERENCES}

[1] M. H. Albadi and E. F. El-Saadany, "Demand response in electricity markets: An overview," in Power Engineering Society General Meeting, 2007. IEEE. IEEE, 2007, pp. 1-5.

[2] G. De Zotti, S. A. P. Kani, J. Morales, H. Madsen, and N. K. Poulsen, "Consumers' flexibility estimation at the TSO level for balancing services," IEEE Transactions on Power Systems, 2018.

[3] M. e. a. V., "Optimization of market clearing price using firefly and PSO algorithm in bilateral electricity market," TENCON 2017- 2017 IEEE Region 10 Conference. IEEE. 2017, pp. 245-249, 2017.

[4] H. Madsen, J. Parvizi, R. Halvgaard, L. E. Sokoler, J. B. Jørgensen, L. H. Hansen, and K. B. Hilger, "Control of electricity loads in future electric energy systems," Handbook of Clean Energy Systems, pp. 1-26, 2015.

[5] G. De Zotti, S. A. P. Kani, J. M. Morales, H. Madsen, and N. K. Poulsen, "A control-based method to meet TSO and DSO ancillary services needs by flexible end-users," IEEE Transactions on Power Systems, 2019.

[6] G. De Zotti, S. A. Pourmousavi, H. Madsen, and N. K. Poulsen, "Ancillary services 4.0: A top-to-bottom control-based approach for solving ancillary service problems in smart grids," IEEE Access, vol. 6, pp. 11 694-706, 2018.

[7] J. M. Morales, A. J. Conejo, H. Madsen, P. Pinson, and M. Zugno, Integrating renewables in electricity markets: operational problems. Springer Science \& Business Media, 2013, vol. 205.

[8] V. Bianco, O. Manca, and S. Nardini, "Electricity consumption forecasting in italy using linear regression models," Energy, vol. 34, no. 9, pp. 1413-1421, 2009.

[9] N. Amjady and M. Hemmati, "Energy price forecasting-problems and proposals for such predictions," IEEE Power and Energy Magazine, vol. 4, no. 2, pp. 20-29, 2006.

[10] F. J. Nogales, J. Contreras, A. J. Conejo, and R. Espínola, "Forecasting next-day electricity prices by time series models," IEEE Transactions on power systems, vol. 17, no. 2, pp. 342-348, 2002.

[11] K. Meng, Z. Dong, and K. Wong, "Self-adaptive radial basis function neural network for short-term electricity price forecasting," IET generation, transmission \& distribution, vol. 3, no. 4, pp. 325-335, 2009.

[12] D. Singhal and K. Swarup, "Electricity price forecasting using artificial neural networks," International Journal of Electrical Power \& Energy Systems, vol. 33, no. 3, pp. 550-555, 2011.

[13] R. Gareta, L. M. Romeo, and A. Gil, "Forecasting of electricity prices with neural networks," Energy conversion and management, vol. 47, no. 13-14, pp. 1770-1778, 2006.
[14] Energinet, "Energinet," https://energinet.dk, 2019, accessed: 2019-01-04.

[15] "Små prosumeres samspil med fremtidens energisystem i 2030," https://energinet.dk, 2018, accessed: 2019-05-18.

[16] Energinet, "SIFRE simulation of flexible and renewable energy resources," https://energinet.dk/analyse-og-forskning/beregningsmodeller, 2015, accessed: 2018-14-12.

[17] S. Chen, S. Billings, and P. Grant, "Non-linear system identification using neural networks," International journal of control, vol. 51, no. 6 , pp. 1191-1214, 1990.

[18] S. N. Kumpati et al., "Identification and control of dynamical systems using neural networks," IEEE Transactions on Neural Networks, 1990.

[19] V. Topuz, "Hourly traffic flow predictions by different ann models," Urban Transport and Hybrid Vehicles, p. 192, 2010.

[20] M. Nielsen, "Using neural nets to recognize handwritten digits," Neural Networks and Deep Learning, 2015.

[21] P. Zhou and J. Austin, "Learning criteria for training neural network classifiers," Neural computing \& applications, vol. 7, no. 4, pp. 334342, 1998.

[22] P. Golik, P. Doetsch, and H. Ney, "Cross-entropy vs. squared error training: a theoretical and experimental comparison." in Interspeech, vol. 13, 2013, pp. 1756-1760.

[23] M. Kayri, "Predictive abilities of Bayesian regularization and Levenberg-Marquardt algorithms in artificial neural networks: a comparative empirical study on social data," Mathematical and Computational Applications, vol. 21, no. 2, p. 20, 2016.

[24] M. Ranjbar, S. Soleymani, N. Sadati, and A. Ranjbar, "Electricity price forecasting using artificial neural network," in Power Electronics, Drives and Energy Systems, 2006. PEDES'06. International Conference on. IEEE, 2006, pp. 1-5. 